\title{
The SYGMA programme of phase 3 trials to evaluate the efficacy and safety of budesonide/formoterol given 'as needed' in mild asthma: study protocols for two randomised controlled trials
}

\author{
Paul M. O'Byrne ${ }^{1,9^{*}}$, J. Mark FitzGerald ${ }^{2}$, Nanshan Zhong ${ }^{3}$, Eric Bateman ${ }^{4}$, Peter J. Barnes ${ }^{5}$, Christina Keen ${ }^{6}$,
} Gun Almqvist ${ }^{6}$, Kristine Pemberton ${ }^{7}$, Carin Jorup $^{6}$, Stefan Ivanov ${ }^{6}$ and Helen K. Reddel ${ }^{8}$

\begin{abstract}
Background: In many patients with mild asthma, the low frequency of symptoms and the episodic nature of exacerbations make adherence to regular maintenance treatment difficult. This often leads to over-reliance on short-acting $\beta_{2}$-agonist (SABA) reliever medication and under-treatment of the underlying inflammation, with poor control of asthma symptoms and increased risk of exacerbations. The use of budesonide/formoterol 'as needed' in response to symptoms may represent an alternative treatment option for patients with mild asthma.

Methods/design: The SYmbicort Given as needed in Mild Asthma (SYGMA) programme consists of two 52-week, double-blind, randomised, multicentre, parallel-group, phase 3 trials of patients aged 12 years and older with a clinical diagnosis of asthma for at least 6 months, who would qualify for treatment with regular inhaled corticosteroids (ICS). SYGMA1 aims to recruit 3750 patients who will be randomised to placebo twice daily (bid) plus as-needed budesonide/formoterol 160/4.5 $\mu \mathrm{g}$, placebo bid plus as-needed terbutaline $0.4 \mathrm{mg}$, or budesonide $200 \mu \mathrm{g}$ bid plus as-needed terbutaline $0.4 \mathrm{mg}$. The primary objective is to demonstrate the superiority of as-needed budesonide/formoterol over as-needed terbutaline for asthma control, as measured by well-controlled asthma weeks; a secondary objective is to establish the noninferiority of as-needed budesonide/formoterol versus maintenance budesonide plus as-needed terbutaline using the same outcome measure. SYGMA2 aims to recruit 4114 patients who will be randomised to placebo bid plus as-needed budesonide/formoterol 160/4.5 $\mu \mathrm{g}$, or budesonide $200 \mu \mathrm{g}$ bid plus as-needed terbutaline $0.4 \mathrm{mg}$. The primary objective is to demonstrate the noninferiority of as-needed budesonide/formoterol over budesonide bid plus as-needed terbutaline as measured by the annualised severe exacerbation rate. In both studies, use of all blinded study inhalers will be recorded electronically using Turbuhaler ${ }^{\oplus}$ Usage Monitors.

(Continued on next page)
\end{abstract}

\footnotetext{
* Correspondence: obyrnep@mcmaster.ca

${ }^{1}$ Michael G DeGroote School of Medicine, Faculty of Health Sciences,

McMaster University, Hamilton, ON, Canada

${ }^{9}$ Firestone Institute of Respiratory Health, St Joseph's Healthcare and

Department of Medicine, McMaster University, 1280 Main Street West,

Hamilton, ON L8S 4K1, Canada

Full list of author information is available at the end of the article
} 
(Continued from previous page)

Discussion: Given the known risks of mild asthma, and known poor adherence with regular inhaled corticosteroids, the results of the SYGMA programme will help to determine the efficacy and safety of as-needed budesonide/ formoterol therapy in mild asthma. Patient recruitment is complete, and completion of the phase 3 studies is planned in 2017.

Trial registration: ClinicalTrials.gov identifiers: NCT02149199 SYGMA1 and NCT02224157 SYGMA2. Registered on 16 May 2014 and 19 August 2014, respectively.

Keywords: As-needed, Asthma control, Budesonide/formoterol, Exacerbations, Mild asthma, Prn, Rescue inhaler, SYGMA

\section{Background}

Approximately $50-75 \%$ of patients with asthma are considered to have mild disease, but the risks, especially of exacerbations, are often poorly recognised [1]. The longterm goals of asthma management, including mild disease, are to achieve good symptom control and minimise the future risk of exacerbations, fixed airflow limitation and side effects [2]. However, asthma remains uncontrolled in many patients, despite the availability of effective treatment regimens $[1,3]$.

Although inhaled corticosteroids (ICS) are an effective treatment for asthma, poor adherence to prescribed maintenance therapy results in under-treatment of the underlying inflammation and an increased risk of exacerbations, disease progression and death [4-9]. In addition, overreliance on short-acting $\beta_{2}$-agonist (SABA) reliever medication for symptomatic improvement often leads to a delay in the introduction of ICS in patients with mild asthma [10]. Consequently, an alternative approach could be to use an ICS in combination with either a SABA or a rapid- and long-acting $\beta_{2}$-agonist (LABA) as reliever medication without concurrent maintenance treatment, ensuring that ICS is delivered whenever the patient experiences asthma symptoms, and allowing titration of both ICS, and SABA or LABA, according to patient need.

There is evidence to suggest that a combination of ICS and a rapid-acting $\beta_{2}$-agonist is effective when given as needed' in response to asthma symptoms, across all disease severities. In patients with mild asthma, the BEST study showed that the as-needed use of beclometasone dipropionate (BDP) and salbutamol in a single inhaler was noninferior to regular ICS maintenance therapy [11]. For mild-to-moderate asthma, another study has shown that the time to treatment failure was similar when patients received as-needed BDP and salbutamol in separate inhalers compared with a maintenance dose of ICS adjusted at 6weekly intervals based on physician assessment [12]. Similarly, as-needed budesonide/formoterol was shown to reduce fractional exhaled nitric oxide ( $\mathrm{FeNO}$ ) and improve lung function compared with as-needed formoterol in patients with so-called intermittent asthma and elevated baseline FeNO [13]. In moderate-to-severe asthma, asneeded budesonide/formoterol used in addition to maintenance therapy reduced the rate of severe exacerbations compared with either formoterol or terbutaline reliever therapy, as shown in the Symbicort SMART ${ }^{\mathrm{max}}$ programme [14]. Consequently, there is a need for largescale randomised clinical trials to further assess this asneeded approach in patients with mild asthma, as highlighted in recent reviews $[15,16]$.

The SYmbicort Given as needed in Mild Asthma (SYGMA) programme aims to evaluate the efficacy and safety of as-needed budesonide/formoterol in patients with mild asthma. Here, we describe the rationale and design of two ongoing 52-week phase 3 trials (SYGMA1 [NCT02149199, registered on 16 May 2014] and SYGMA2 [NCT02224157, registered on 19 August 2014]) that are comparing as-needed budesonide/formoterol with either maintenance budesonide plus as-needed terbutaline, or asneeded terbutaline alone. Asthma symptom control [2], as assessed by well-controlled asthma weeks, and annual severe exacerbation rate are being investigated as primary endpoints. Secondary endpoints include: average change from baseline in pre-dose forced expiratory volume in $1 \mathrm{~s}$ $\left(\mathrm{FEV}_{1}\right)$, Asthma Control Questionnaire (ACQ-5) score, health-related quality of life, asthma symptom control, medication intake measured via the Turbuhaler ${ }^{\circ}$ Usage Monitor (TUM), the percentage of controller-use days, and adverse events (AEs).

\section{Methods/design \\ Patients}

Patients meeting the inclusion and exclusion criteria as detailed in Table 1 were eligible for inclusion in the SYGMA programme. The same inclusion and exclusion criteria apply to both SYGMA1 and SYGMA2.

In brief, patients were eligible for inclusion if they were aged 12 years or older at visit 1 (Table 2, Figs. 1 and 2), with a documented clinical diagnosis of asthma according to Global Initiative for Asthma (GINA) criteria [17] for at least 6 months prior to visit 1 and with confirmation of diagnosis by bronchodilator reversibility. Lung function and reversibility tests performed as part of visits 2 and 3 can be used as confirmation of asthma diagnosis if there is no measure of lung function available before visit 1 . Patients must have evidence of need 
Table 1 Key inclusion and exclusion criteria for participation in the SYGMA programme

\begin{tabular}{|c|c|}
\hline Key inclusion criteria & Key exclusion criteria \\
\hline 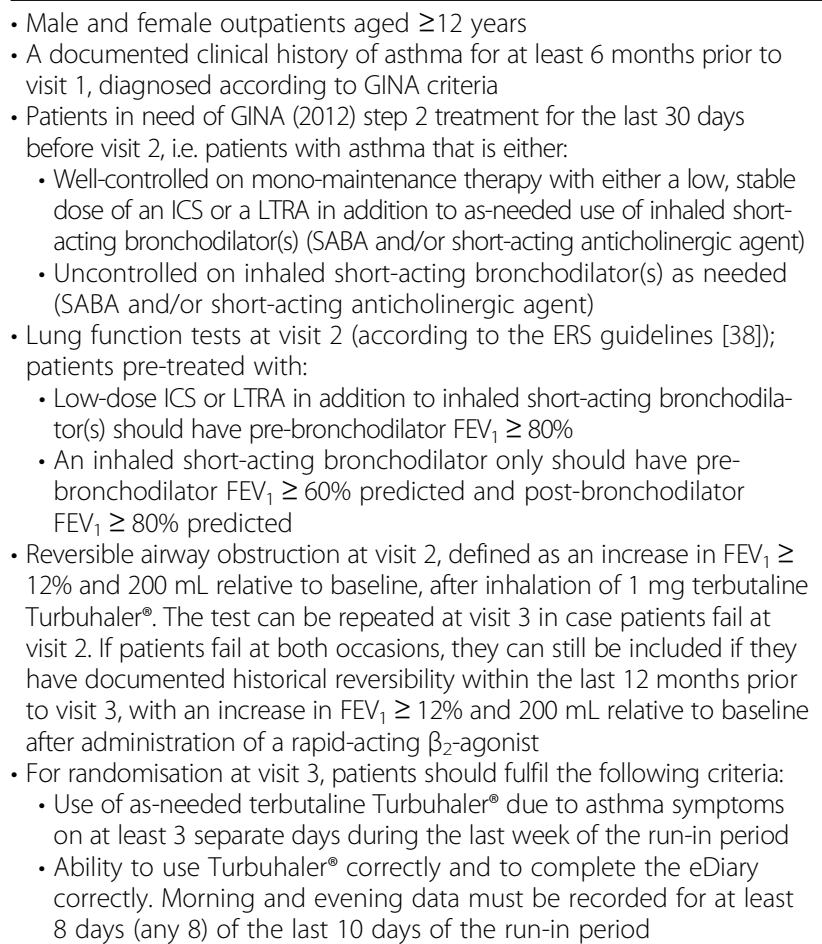 & $\begin{array}{l}\text { - Any asthma worsening requiring change in asthma treatment } \\
\text { other than SABA and/or short-acting anticholinergic agent } \\
\text { within } 30 \text { days prior to visit } 1 \text { or during run-in } \\
\text { - Use of oral, rectal or parenteral GCS within } 30 \text { days and/or } \\
\text { depot parenteral GCS within } 12 \text { weeks prior to visit } 1 \\
\text { - Smoker (current or previous) with a smoking history of } \geq 10 \\
\text { pack years } \\
\text { - Use of any } \beta \text {-blocking agent, including eye drops } \\
\text { - Any significant disease or disorder (e.g. cardiovascular, } \\
\text { gastrointestinal, hepatic, renal) } \\
\text { - Pregnancy, breast-feeding or planned pregnancy } \\
\text { - For randomisation at visit } 3 \text {, patients should not fulfil any of the } \\
\text { following criteria: } \\
\text { - Use of } \geq 6 \text { terbutaline Turbuhaler }{ }^{\otimes} \text { as-needed inhalations per } \\
\text { day, for a certain number of days depending on the actual } \\
\text { length of run-in: for } \geq 2 \text { days out of } 14 \text { days; for } \geq 3 \text { days out } \\
\text { of } 15-21 \text { days; for } \geq 4 \text { days out of } 22 \text { or more days of run-in }\end{array}$ \\
\hline
\end{tabular}

eDiary electronic diary, ERS European Respiratory Society, FEV , forced expiratory volume in $1 \mathrm{~s}$, GCS glucocorticosteroid, GINA Global Initiative for Asthma, ICS inhaled corticosteroid, LTRA leukotriene receptor antagonist, SABA short-acting $\beta_{2}$-agonist

for GINA step 2 treatment (low-dose ICS) for the 30 days before visit 2, either by asthma being wellcontrolled on mono-maintenance therapy with a low stable dose of an ICS or a leukotriene receptor antagonist (LTRA) in addition to as-needed short-acting bronchodilator (SABA and/or short-acting anticholinergic agent) (subgroup 1); or by asthma being uncontrolled on as-needed, inhaled short-acting bronchodilator(s) (subgroup 2). Additional inclusion criteria included baseline pre-bronchodilator $\mathrm{FEV}_{1} \geq 80 \%$ predicted (for subgroup 1 ) or $\geq 60 \%$ predicted (for subgroup 2) (Table 1). Exclusion criteria included a recent asthma exacerbation, history of life-threatening asthma requiring intubation, and current or past smokers with a smoking history of at least 10 pack-years.

In both studies, patients who fulfilled all the inclusion and none of the exclusion criteria at visit 2 entered a 2-4week run-in period in order to demonstrate the appropriateness of candidates prior to randomisation (Figs. 1 and 2). Patients were to stop any prescribed asthma medication (including SABA and maintenance treatment with ICS or LTRA) that was being used at the time of study entry, and all patients were to receive as-needed Bricanyl ${ }^{\circ}$ Turbuhaler ${ }^{\oplus} 0.5 \mathrm{mg}$ during run-in (which corresponds to the blinded terbutaline Turbuhaler $0.4 \mathrm{mg}$, with regards to the dose delivered). At visit 3, patients will be randomised if they required as-needed terbutaline for the relief of asthma symptoms on at least three separate days during the last week of the run-in period; however, patients will be excluded if, during run-in, they used asneeded terbutaline six or more times per day on: at least 2 days out of 14 days; at least 3 days out of 15-21 days; at least 4 days out of 22 or more days (depending on the duration of run-in). All study medication, including terbutaline during run-in, will be delivered via Turbuhaler ${ }^{\ominus}$ and recorded electronically using the TUM [18].

The study is being performed in accordance with the Declaration of Helsinki and Good Clinical Practice. The protocols for the SYGMA 1 and SYGMA 2 trials have been approved by the oversight authorities in the countries detailed in Additional file 1: Table S1. All patients were required to provide written informed consent before participating in the programme, and recruitment was not to begin in an individual site before all local approvals had been obtained.

\section{SYGMA1 study design}

Target recruitment for SYGMA1 is 3750 patients from approximately 18 countries. These patients will be randomised to either placebo twice daily (bid) plus asneeded budesonide/formoterol $160 / 4.5 \mu \mathrm{g}$, placebo bid plus as-needed terbutaline $0.4 \mathrm{mg}$, or budesonide $200 \mu \mathrm{g}$ 
Table 2 Standard Protocol Items: Recommendations for Interventional Trials (SPIRIT) figures for SYGMA1 (A) and SYGMA2 (B)

\begin{tabular}{|c|c|c|c|c|c|c|c|c|c|}
\hline & Enrolment & Run-in & Randomisation & Treatment & & & & & Follow-up \\
\hline \multicolumn{10}{|l|}{$\bar{A}$} \\
\hline Week & & -2 to -4 & 0 & 4 & 16 & 28 & 40 & 52 & 54 \\
\hline Visit & 1 & 2 & 3 & 4 & 5 & 6 & 7 & 8 & Phone \\
\hline Informed consent & $x$ & & & & & & & & \\
\hline Allocation of enrolment code (IVRS/IWRS) & $x$ & & & & & & & & \\
\hline Demography (date of birth, gender, race) & $x$ & & & & & & & & \\
\hline Inclusion/exclusion criteria & $x$ & $x$ & $x$ & & & & & & \\
\hline Medical, surgical history & $x$ & & & & & & & & \\
\hline $\begin{array}{l}\text { Asthma history (including history of severe } \\
\text { asthma exacerbations) }\end{array}$ & $x$ & & & & & & & & \\
\hline Smoking history & $x$ & & & & & & & & \\
\hline $\begin{array}{l}\text { Patient training in eDiary, Turbuhaler } \\
\text { (inhalation technique), TUM and PEF meter use }\end{array}$ & & $x$ & & & & & & & \\
\hline ACQ-5 and AQLQ(S) at study site & & $x$ & $x$ & Only ACQ-5 & $x$ & $x$ & $x$ & $x$ & \\
\hline SAEs (from visit 1)/AEs (from visit 2) & $x$ & $x$ & $x$ & $x$ & $x$ & $x$ & $x$ & $x$ & $x$ \\
\hline $\begin{array}{l}\text { Weight and height (height only for adolescents at } \\
\text { visit 8) }\end{array}$ & & $x$ & & & & & & $x$ & \\
\hline Physical examination & & $x$ & & & & & & $x$ & \\
\hline Pulse and blood pressure & & $x$ & & & & & & $x$ & \\
\hline Pregnancy test & & $x$ & & & & & & & \\
\hline Adjustment of current asthma medication & & $x$ & & & & & & & \\
\hline Randomisation & & & $x$ & & & & & & \\
\hline Bricany $\left.\right|^{\circledast}$ for run-in dispense $[d] /$ return $[r]$ & & $d$ & r & & & & & & \\
\hline $\begin{array}{l}\text { Lung function (FEV }{ }_{1}, F V C \text { pre and post Bricanyl }\left.\right|^{\oplus} \\
\text { administration) }\end{array}$ & & $x$ & $x$ & $x$ & $x$ & $x$ & $x$ & $x$ & \\
\hline $\begin{array}{l}\text { Reversibility test (calculated at visit } 2 \text { and if } \\
\text { needed, calculated at visit } 3 \text { as well) }\end{array}$ & & $x$ & $x$ & & & & & & \\
\hline Concomitant medication & & $x$ & $x$ & $x$ & $x$ & $x$ & $x$ & $x$ & \\
\hline $\begin{array}{l}\text { Investigational product (dispense }[\mathrm{d}] / \text { /return }[r] / \\
\text { check }[c] \text { ) }\end{array}$ & & & $d$ & $d / r / c$ & $d / r / c$ & $d / r / c$ & $d / r / c$ & $r / c$ & \\
\hline $\begin{array}{l}\text { Intake of maintenance treatment morning } \\
\text { dose }\end{array}$ & & & $x$ & $x$ & $x$ & $x$ & $x$ & & \\
\hline $\begin{array}{l}\text { Review of PEF, asthma symptoms, night-time } \\
\text { awakenings, maintenance and 'as needed' IP in- } \\
\text { take and Turbuhaler }{ }^{\otimes} \text { user technique; re-training } \\
\text { of patient if needed }\end{array}$ & & & $x$ & $x$ & $x$ & $x$ & $x$ & $x$ & \\
\hline Review of patient's compliance with eDiary & & & $x$ & $x$ & $x$ & $x$ & $x$ & $x$ & \\
\hline Informed consent (qualitative substudy) & & & & $x^{a}$ & $x^{a}$ & $X^{a}$ & $x^{a}$ & & \\
\hline \multicolumn{10}{|l|}{ B } \\
\hline Week & & -2 to -4 & 0 & 8 & 17 & 25 & 34 & $52^{b}$ & 54 \\
\hline Visit & 1 & 2 & 3 & Phone & & Phone & & Phone & \\
\hline Written informed consent & $x$ & & & & & & & & \\
\hline Allocation of enrolment code & $x$ & & & & & & & & \\
\hline Demography & $x$ & & & & & & & & \\
\hline Inclusion/exclusion criteria & $x$ & $x$ & $x$ & & & & & & \\
\hline Medical/surgical history & $x$ & & & & & & & & \\
\hline Asthma history (including exacerbation history) & $x$ & & & & & & & & \\
\hline
\end{tabular}


Table 2 Standard Protocol Items: Recommendations for Interventional Trials (SPIRIT) figures for SYGMA1 (A) and SYGMA2 (B) (Continued)

\begin{tabular}{|c|c|c|c|c|c|c|c|c|}
\hline Smoking history & $x$ & & & & & & & \\
\hline ACQ-5, AQLQ(S) & & $x$ & $x$ & & $x$ & $x$ & $x$ & \\
\hline $\begin{array}{l}\text { Health Care resource utilisation questionnaire, } \\
\text { EQ-5D-5L }\end{array}$ & & & $x$ & & $x$ & $x$ & $x$ & \\
\hline $\mathrm{SAE} / \mathrm{AES} \mathrm{s}^{\mathrm{C}}$ & $x^{c}$ & $x^{c}$ & $x$ & & $x$ & $x$ & $x$ & $x$ \\
\hline Weight and height & & $x$ & & & & & $X^{d}$ & \\
\hline Physical examination & & $x$ & & & & & $x$ & \\
\hline Vital signs (pulse and blood pressure) & & $x$ & & & & & $x$ & \\
\hline Pregnancy test (if applicable) & & $x$ & & & & & & \\
\hline Adjustment of current asthma medication & & $x$ & & & & & & \\
\hline $\begin{array}{l}\text { Patient training in how to use Turbuhaler } \\
\text { (inhalation technique) and TUM }\end{array}$ & & $x$ & & & & & & \\
\hline Bricany $\|^{\oplus}$ for run- in (dispense $[\mathrm{d}] /$ return $[\mathrm{r}]$ ) & & $d$ & r & & & & & \\
\hline Randomisation & & & $x$ & & & & & \\
\hline $\begin{array}{l}\text { Lung function (FEV }{ }_{1}, \text { FVC pre and post Bricanyl }{ }^{\circledR} \\
\text { administration) }\end{array}$ & & $x$ & $x$ & & $x$ & $x$ & $x$ & \\
\hline Reversibility test ${ }^{\mathrm{e}}$ & & $x$ & $x^{e}$ & & & & & \\
\hline Collection of severe asthma exacerbations & & & & $x^{f}$ & $x^{f} \quad x^{f}$ & $x^{f}$ & $x^{f}$ & \\
\hline Concomitant medications & & $x$ & $x$ & & $x$ & $x$ & $x$ & \\
\hline $\begin{array}{l}\text { Investigational product (dispense [d]/return [r]/ } \\
\text { check [c]) }\end{array}$ & & & $d$ & & $\mathrm{~d} / \mathrm{r} / \mathrm{c}$ & $\mathrm{d} / \mathrm{r} / \mathrm{c}$ & $r / c$ & \\
\hline
\end{tabular}

\section{Notes:}

ACQ-5, Asthma Control Questionnaire 5 questions; AE, adverse event; AQLQ(S), Standardised Asthma Quality of Life Questionnaire; EQ-5D-5L, EuroQol 5-dimensions 5level; FEV 1 , forced expiratory volume in $1 \mathrm{~s}$; FVC, forced vital capacity; IP, investigational product; IWRS/IVRS, Interactive Web and Voice Response System; PEF, morning peak expiratory flow; SAE, serious adverse event; TUM, Turbuhaler ${ }^{\oplus}$ Usage Monitor, recording use of each blinded study inhalers

${ }^{a}$ Obtaining informed consent of patients into the qualitative substudy is only applicable to the subset of sites selected to participate. Informed consent into the substudy is to be obtained before any interview-related activities. Informed consent can occur at any time at visit 4 (week 4 ) or later; however, the qualitative patient interview conducted with the patient will occur between week 12 and week 50 for each patient who has elected to participate. The exact time point of the interview will be determined by the contract research organisation

${ }^{\mathrm{b}}$ After discontinuation of an investigational drug (ie before visit 6) patients will be followed up according to the original visit schedule including site visits and phone contacts. Only severe asthma exacerbations, AEs and concomitant medications will be collected. If it is not possible for the patient to visit the study site, the visit(s) may be performed via phone

'Serious adverse events will be collected from the time of signing informed consent. Adverse events will be collected from visit 2

${ }^{\mathrm{d}}$ Height only for adolescents

${ }^{e}$ Reversibility test will be performed at visit 2 . The test can be repeated at visit 3 in case the patients fail to meet the inclusion criterion at visit 2

fSevere asthma exacerbations will be collected from visit 3 through the entire study

bid plus as-needed terbutaline $0.4 \mathrm{mg}$ (Fig. 1). Additional short-term $(2-4$ weeks $)$ treatment with open-label inhaled budesonide $200 \mu \mathrm{g}$ bid is allowed for patients experiencing moderate or severe asthma exacerbations or having long-term poor asthma control. After this time, the patient should be evaluated and 'stepped down' to blinded study treatment if possible. If patients require additional budesonide treatment on two separate occasions, either for a moderate exacerbation or for longterm poorly-controlled asthma, the investigator should consider continuing with additional budesonide for the rest of the study. Weeks with open-label budesonide use are not defined as well-controlled asthma weeks. Patients will be instructed to contact the investigator if using more than 12 inhalations/day of as-needed budesonide/formoterol or terbutaline, or if they feel that they are in need of medical assistance. An electronic diary
(eDiary) will also alert patients to contact their study physician if symptoms are increasing, and/or if their lung function deteriorates as measured by morning peak expiratory flow (PEF). In addition, all patients will be closely monitored during the course of the study with treatment visits taking place at 4, 16, 28, 40, and 52 weeks after randomisation (Table $2 \mathrm{a}$ ).

\section{SYGMA2 study design}

Target recruitment for SYGMA2 is 4114 patients from approximately 25 countries. These patients will be randomised to either placebo bid plus as-needed budesonide/formoterol 160/4.5 $\mu \mathrm{g}$ or budesonide $200 \mu \mathrm{g}$ bid plus as-needed terbutaline $0.4 \mathrm{mg}$ (Fig. 2). Patients will be instructed to contact the investigator if using more than 12 inhalations/day of as-needed budesonide/ 


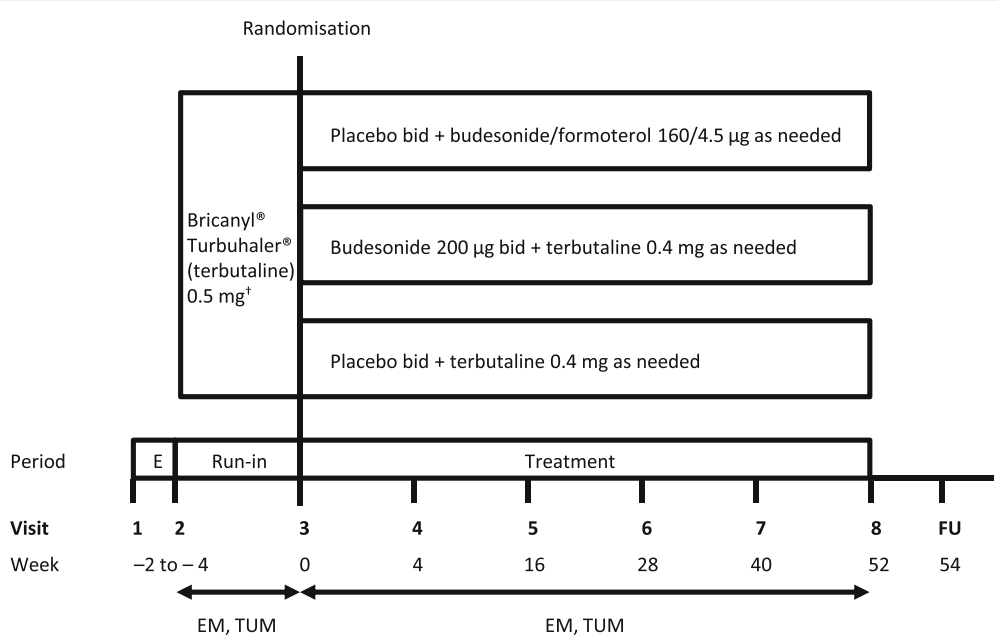

Fig. 1 SYGMA1 study design. bid twice daily, E enrolment, EM electronic monitoring twice daily (eDiary), FU follow-up phone call, TUM Turbuhaler ${ }^{\circledR}$ Usage Monitor, recording use of each blinded study inhalers. ${ }^{\dagger}$ Corresponds to the terbutaline Turbuhaler ${ }^{\oplus} 0.4 \mathrm{mg}$, with regards to the dose delivered

formoterol or terbutaline, or if they feel that they are in need of medical assistance.

Treatment visits will take place at 17, 34, and 52 weeks after randomisation. Between site visits, study staff will contact patients by phone to record additional asthma treatment and hospitalisation/emergency treatment due to asthma (including severe asthma exacerbation data; Table 2b).

\section{Efficacy assessments}

Details of the primary and secondary efficacy endpoints included in the SYGMA1 and SYGMA2 studies are presented in Table 3. In SYGMA1, the primary objective is to demonstrate that as-needed budesonide/formoterol is superior to as-needed terbutaline in terms of asthma control as measured by well-controlled asthma weeks [19], achieved when two or more of the following criteria are fulfilled: no more than 2 days with a daily asthma symptom score $>1$; no more than 2 days of as-needed medication use, up to a maximum of four occasions per week (multiple occasions per day are regarded as separate occasions); morning PEF $\geq 80 \%$ predicted every day. Both of the following criteria must also be fulfilled: no nighttime awakenings due to asthma; no additional inhaled and/or systemic corticosteroid treatment due to asthma [19]. A secondary objective is to establish the noninferiority of as-needed budesonide/formoterol versus maintenance budesonide plus as-needed terbutaline using the same outcome measure. The primary objective of SYGMA2 is to demonstrate that as-needed budesonide/ formoterol is noninferior to twice-daily budesonide plus as-needed terbutaline at reducing the annual severe asthma exacerbation rate (Table 3). Secondary efficacy and safety outcome measures are shown in Table 3.

In both SYGMA1 and SYGMA2, a severe asthma exacerbation is defined using American Thoracic Society/

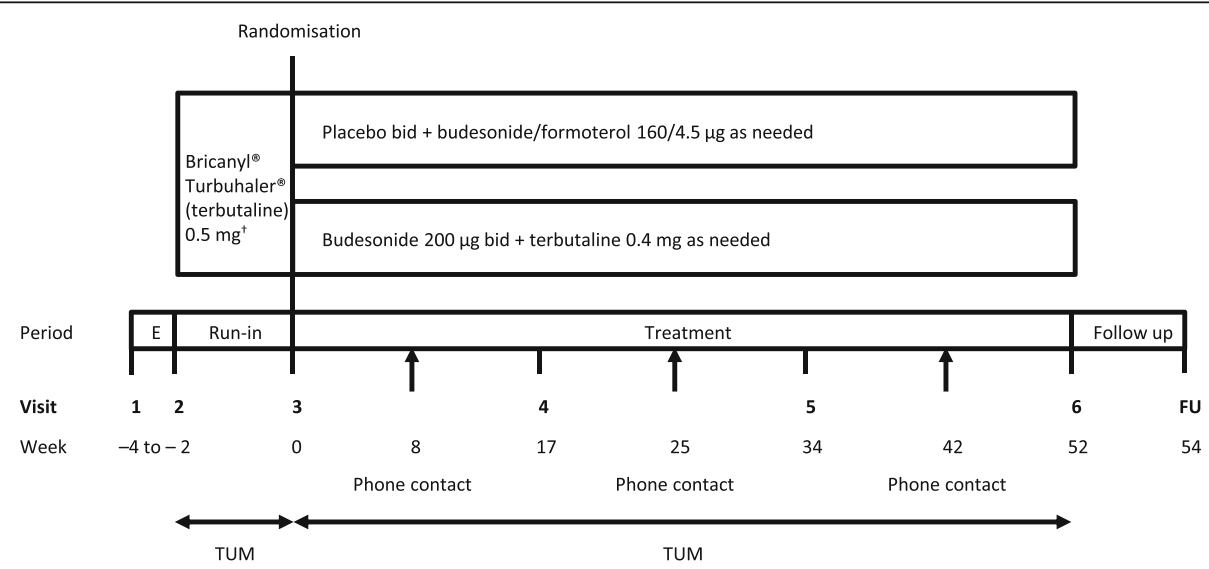

Fig. 2 SYGMA2 study design. bid twice daily, E enrolment, FU follow-up phone call, TUM Turbuhaler ${ }^{\otimes}$ Usage Monitor, recording use of each blinded study inhalers. ${ }^{\dagger}$ Corresponds to the terbutaline Turbuhaler $0.4 \mathrm{mg}$, with regards to the dose delivered 
Table 3 Endpoints of the SYGMA programme

\begin{tabular}{|c|c|c|c|c|}
\hline & \multicolumn{2}{|l|}{ SYGMA1 } & \multicolumn{2}{|l|}{ SYGMA2 } \\
\hline & Comparator & Outcome measure & Comparator & Outcome measure \\
\hline $\begin{array}{l}\text { Primary } \\
\text { endpoints }\end{array}$ & $\begin{array}{l}\text { As-needed budesonide/formoterol vs } \\
\text { as-needed terbutaline }\end{array}$ & $\begin{array}{l}\text { - Evaluation of asthma } \\
\text { control as measured by } \\
\text { well-controlled asthma } \\
\text { weeks }\end{array}$ & $\begin{array}{l}\text { As-needed budesonide/ } \\
\text { formoterol vs budesonide } \\
\text { bid plus as-needed } \\
\text { terbutaline }\end{array}$ & $\begin{array}{l}\text { - Annual severe asthma } \\
\text { exacerbation rate }\end{array}$ \\
\hline \multirow[t]{2}{*}{$\begin{array}{l}\text { Secondary } \\
\text { efficacy } \\
\text { endpoints }\end{array}$} & $\begin{array}{l}\text { As-needed budesonide/formoterol vs } \\
\text { budesonide bid plus as-needed } \\
\text { terbutaline }\end{array}$ & $\begin{array}{l}\text { - Evaluation of asthma } \\
\text { control as measured by } \\
\text { well-controlled asthma } \\
\text { weeks }\end{array}$ & $\begin{array}{l}\text { As-needed budesonide/ } \\
\text { formoterol vs budesonide } \\
\text { bid plus as-needed } \\
\text { terbutaline }\end{array}$ & \multirow[b]{2}{*}{$\begin{array}{l}\text { - Time to first severe asthma } \\
\text { exacerbation } \\
\text { - Average change from } \\
\text { baseline in pre-dose FEV } 1 \\
\text { - Time to study specific } \\
\text { asthma-related } \\
\text { discontinuation } \\
\text { - Average change from } \\
\text { baseline in number of } \\
\text { inhalations of as-needed } \\
\text { medication } \\
\text { - Change from baseline in } \\
\text { percent of as-needed free } \\
\text { days } \\
\text { - Percentage of controller use } \\
\text { days } \\
\text { - Average change from } \\
\text { baseline in ACQ-5 score and } \\
\text { responders based on MID } \\
\text { - Average change from } \\
\text { baseline in AQLQ score } \\
\text { - Total ICS load and number } \\
\text { of days with systemic } \\
\text { corticosteroid treatment }\end{array}$} \\
\hline & $\begin{array}{l}\text { As-needed budesonide/formoterol vs } \\
\text { as-needed terbutaline or budesonide } \\
\text { bid plus as-needed terbutaline }\end{array}$ & $\begin{array}{l}\text { - Time to first severe asthma } \\
\text { exacerbation } \\
\text { - Time to first moderate or } \\
\text { severe asthma exacerbation } \\
\text { - Average change from } \\
\text { baseline in pre-dose FEV }{ }_{1} \\
\text { - Average change from } \\
\text { baseline in morning and } \\
\text { evening PEF } \\
\text { - Average change from } \\
\text { baseline in number of } \\
\text { inhalations of as-needed } \\
\text { medication } \\
\text { - Average change from } \\
\text { baseline in symptom score } \\
\text { - Percentage of night-time } \\
\text { awakenings due to asthma } \\
\text { - Percentage of symptom- } \\
\text { free days } \\
\text { - Percentage of as-needed } \\
\text { free days } \\
\text { - Percentage of asthma } \\
\text { control days } \\
\text { - Percentage of controller use } \\
\text { days } \\
\text { - Time to asthma-related } \\
\text { discontinuation } \\
\text { - Poorly-controlled asthma } \\
\text { weeks } \\
\text { - Time to additional steroids } \\
\text { for asthma } \\
\text { - Average change from } \\
\text { baseline in ACQ-5 score and } \\
\text { responders based on MID } \\
\text { - Average change from } \\
\text { baseline in AQLQ score } \\
\text { - Total ICS load and number } \\
\text { of days with systemic } \\
\text { corticosteroid treatment }\end{array}$ & & \\
\hline $\begin{array}{l}\text { Safety } \\
\text { endpoints }\end{array}$ & $\begin{array}{l}\text { As-needed budesonide/formoterol vs } \\
\text { as-needed terbutaline or budesonide } \\
\text { bid plus as-needed terbutaline }\end{array}$ & $\begin{array}{l}\text { - Adverse events (nature, } \\
\text { incidence and severity) } \\
\text { - Pulse, blood pressure and } \\
\text { physical examination }\end{array}$ & $\begin{array}{l}\text { As-needed budesonide/ } \\
\text { formoterol vs budesonide } \\
\text { bid plus as-needed } \\
\text { terbutaline }\end{array}$ & $\begin{array}{l}\text { - Adverse events (nature, } \\
\text { incidence and severity) } \\
\text { - Pulse, blood pressure and } \\
\text { physical examination }\end{array}$ \\
\hline $\begin{array}{l}\text { Exploratory } \\
\text { endpoints }\end{array}$ & N/A & $\begin{array}{l}\text { - Coded transcriptions of } \\
\text { patient interviews }\end{array}$ & $\begin{array}{l}\text { As-needed budesonide/ } \\
\text { formoterol vs budesonide } \\
\text { bid plus as-needed } \\
\text { terbutaline }\end{array}$ & $\begin{array}{l}\text { - EuroQol 5-dimensional 5- } \\
\text { level questionnaire } \\
\text { - Health Economics } \\
\text { Questionnaire for resource } \\
\text { utilisation }\end{array}$ \\
\hline
\end{tabular}

ACQ-5 Asthma Control Questionnaire 5-item version, AQLQ Asthma Quality of Life Questionnaire standard version, bid twice daily, FEV ${ }_{1}$ forced expiratory volume in $1 \mathrm{~s}$, ICS inhaled corticosteroid, MID minimal important difference, PEF peak expiratory flow

Daily asthma symptom score $=$ the sum of the morning and evening symptom score

European Respiratory Society (ATS/ERS) Task Force criteria [20] as worsening of asthma that is associated with a medical intervention, requiring either the use of systemic corticosteroids for at least 3 days (or an injection of depot corticosteroids), or inpatient hospitalisation or an emergency department (ED) visit (or other 
urgent, unscheduled health care visit) due to asthma that required systemic corticosteroids. Moderate exacerbations will also be assessed in SYGMA1, and are defined as a deterioration of asthma requiring a change in treatment, i.e. initiation of open-label ICS, to avoid progression to a severe exacerbation. Spirometry assessments in both studies will be performed according to ATS/ERS guidelines [21] on the day of study visits (SYGMA1: visits 2-8; SYGMA2: visits 2-6).

In SYGMA1 only, patients will complete an eDiary twice daily for PEF, asthma symptoms and night-time awakenings due to asthma symptoms. A symptom-free day is defined as a day and night with no asthma symptoms, and a night with no awakenings due to asthma symptoms. Similarly, an asthma-control day is defined as the fulfilment of all of the following criteria: a day and night with no asthma symptoms; a night with no awakenings due to asthma symptoms; a day and night with no as-needed medication use. Poorly-controlled asthma weeks, a secondary variable in SYGMA1 only, are defined as the documentation of one of the following conditions: at least two consecutive days with night-time awakening due to asthma symptoms; as-needed medication use for symptom relief on at least three occasions per day for at least two consecutive days; additional systemic corticosteroids required for a severe asthma exacerbation. In SYGMA1, ACQ-5 and Standardised Asthma Quality of Life Questionnaires (AQLQ) will be selfadministered within the eDiary at scheduled visits. In SYGMA2, ACQ-5 and AQLQ questionnaires will be selfadministered using the electronic Patient Reported Outcome (ePRO) device at scheduled visits.

Use of as-needed or randomised maintenance treatment, and terbutaline use during run-in, will be recorded using the TUM (SmartTurbo ${ }^{\mathrm{Ts}}$, Adherium, New Zealand) in both studies. The TUM is a validated electronic data logger designed to be attached to a Turbuhaler ${ }^{\circ}[18,22]$. The TUM contains an electronic clock that logs the date and time when the Turbuhaler base grip is rotated back and forth. Use of open-label budesonide will be recorded in the electronic Case Report Form (eCRF). Patients will receive appropriate training for all devices (eDiary, PEF meter, TUM, ePRO device) at visit 2.

\section{Safety assessments}

Safety assessments included in both studies are detailed in Table 3. AEs will be recorded from visit 2, throughout the treatment and follow-up periods, until the last phone follow-up, or last contact. Serious AEs (SAEs) will be recorded from the time of informed consent. All AEs will be recorded in the eCRF. Physical examination, blood pressure, and pulse rate measurements will be performed before run-in (visit 2) and at the end of treatment (SYGMA1: visit 8; SYGMA2: visit 6). Follow-up phone contact for AEs will be performed 2 weeks after completion of study treatment. An independent Adjudication Committee will review any fatal events occurring during the SYGMA studies to determine whether these events were asthma-related.

\section{Exploratory assessments}

In SYGMA1, a qualitative substudy is being conducted in a small subset of patients. The exploratory objective of this substudy is to further evaluate when and why patients use the study medications, using a qualitative interview approach [23]. In SYGMA2, information on health care resource utilisation as well as health status (EuroQol 5dimensions 5-level health survey: EQ-5D-5L) will be collected at randomisation and at each treatment visit to enable the cost-effectiveness of the interventions to be assessed. Patients will be asked about ambulatory-setting or home consultations with specialists, primary care physicians or other health care professionals, and phone consultations with physicians or nurses as well as health care resource use in terms of: ambulance services; ED visits; hospital admissions including intensive care. Patients in paid employment and education will be asked how much time they have missed due to their asthma. The EQ-5D-5 L will assess mobility, self-care, usual activity, pain/discomfort, and anxiety/depression, and will be self-administered using the paper version.

\section{Discontinuations}

Study-specific criteria for discontinuations in SYGMA1 are: a severe asthma exacerbation with a duration of more than 3 weeks; two severe asthma exacerbations within a period of 3 months; or three severe asthma exacerbations in total during the study. Study-specific criteria for discontinuations in SYGMA2 are: a severe asthma exacerbation with duration of more than 3 weeks; three severe asthma exacerbations within a period of 6 months.

\section{Sample size estimates}

In SYGMA1, 3750 patients (625 patients/treatment group/pre-study treatment group) are required to give greater than $95 \%$ power to detect superiority of as-needed budesonide/formoterol compared with as-needed terbutaline and $90 \%$ power to establish noninferiority of asneeded budesonide/formoterol compared with budesonide plus as-needed terbutaline for well-controlled asthma weeks, with a pre-defined noninferiority limit of 0.8 , i.e. the lower $95 \%$ confidence interval (CI) of the odds ratio for budesonide/formoterol versus budesonide plus terbutaline is $\geq 0.8$. Assuming that equal numbers of patients are recruited to each of the subgroups (stratified by prestudy treatment), this sample size also gives $80 \%$ power to detect a difference between as-needed budesonide/formoterol and as-needed terbutaline, and $80 \%$ power to 
establish noninferiority of as-needed budesonide/formoterol compared with budesonide plus as-needed terbutaline, with a pre-defined noninferiority limit of 0.78 .

SYGMA2 was initially powered to demonstrate superiority of as-needed budesonide/formoterol compared with budesonide plus as-needed terbutaline as measured by the annualised severe exacerbation rate; 4114 patients (2057 patients/treatment group) were estimated to be required to achieve $90 \%$ power to detect a difference in annualised severe asthma exacerbation rate between treatments, assuming an exacerbation rate of 0.16 per year among patients treated with budesonide, with a $25 \%$ reduction in risk for patients receiving as-needed budesonide/formoterol. To account for uncertainty over the assumed exacerbation rates as well as the dispersion parameter, the overall exacerbation rate was planned to be monitored during the study in a blinded fashion, allowing for an increase of the sample size by a maximum of $50 \%$. A blinded sample size review of SYGMA 2 , performed according to the study protocol and prior to enrolling the last patient, indicated that there would be adequate power to test a noninferiority hypothesis based on the design and assumptions specified in the protocol. The SYGMA 2 protocol was amended to include a noninferiority test as the primary analysis, using a pre-defined noninferiority limit of 1.2.

\section{Statistical analyses}

The primary outcome measure in SYGMA1, wellcontrolled asthma weeks (as-needed budesonide/formoterol versus as-needed terbutaline (superiority) and asneeded budesonide/formoterol versus maintenance budesonide plus as-needed terbutaline (noninferiority)), will be analysed by a repeated measures logistic regression model with: treatment, pre-study treatment, and region as fixed effects; study week as a categorical time variable; and patient as a random effect. The statistical inference will be based on the estimated odds ratio and corresponding 95\% $\mathrm{CI}$ averaged over the whole randomised treatment period.

In SYGMA2, the primary outcome measure of severe exacerbation rate (as-needed budesonide/formoterol versus maintenance budesonide plus as-needed terbutaline (noninferiority)) will be analysed by a negative binomial regression model with treatment, pre-study treatment group, and region as factors. Annual severe exacerbation rates will be estimated and treatment effect will be expressed as the rate ratio and corresponding one-sided noninferiority interval and two-sided 95\% CI. If noninferiority is achieved, then a test for superiority of as needed budesonide/formoterol versus maintenance budesonide will be performed.

The following secondary efficacy endpoints will be assessed in SYGMA1 only. The moderate-to-severe and severe asthma exacerbation rate will be analysed by a negative binomial regression model with treatment, prestudy treatment, and region as factors, and presented as rate ratios and $95 \%$ CIs. Time to first moderate-tosevere exacerbation and time to administration of additional steroids will be analysed by a Cox proportional hazards model with treatment, pre-study treatment group, and region as factors; hazard ratios (HRs) and 95\% CIs will be estimated. Change in eDiary variables from run-in to the mean value of available data during treatment will be analysed by analysis of covariance (ANCOVA) with treatment, pre-study treatment, and region as factors, and mean values during run-in as a continuous covariate. Least squared means (LSMs) by treatment and differences in LSMs between treatments will be estimated, along with corresponding 95\% CIs. Poorly-controlled asthma weeks will be analysed in the same manner as the primary outcome measure, wellcontrolled asthma weeks.

The remaining secondary efficacy endpoints will be analysed in both SYGMA1 and SYGMA2. Time to first severe asthma exacerbation and time to discontinuation due to asthma-related events will be analysed by a Cox proportional hazards model with treatment, pre-study treatment group, and region as factors; HRs and 95\% CIs will be estimated. The treatment effect and 95\% CI for average change from baseline in pre-dose $\mathrm{FEV}_{1}$, ACQ-5, and AQLQ will be analysed using a mixedmodel repeated measures analysis, with the analysis including terms for treatment, pre-study treatment group, region, visit, and treatment by visit. $\mathrm{FEV}_{1}$ data will be analysed with baseline $\mathrm{FEV}_{1}$ included as a covariate. For ACQ-5 and AQLQ, change from baseline to the end of treatment will also be analysed by ANCOVA with treatment, pre-study treatment group, and region as factors and baseline as a continuous variable. Responder variables, based on minimal important difference for ACQ-5 and AQLQ, will be analysed using a logistic regression model with treatment, region, and pre-study treatment as factors, and baseline as a covariate. From the logistic regression model, treatment effects will be estimated by odds ratio and its corresponding 95\% CI. The percentage of controller-use days and as-needed use will be analysed by ANCOVA with treatment, pre-study treatment group, and region as factors; LSMs by treatment and differences in LSMs between treatments will be estimated along with corresponding 95\% CIs. In SYGMA1, sensitivity analyses will be performed to explore the impact of the individual components of well-controlled asthma weeks, paying specific attention to the as-needed component. In SYGMA2, a sensitivity analysis for the primary variable will include all data for patients who discontinue study medication but remain in the study.

For both studies, AEs will be listed for each patient and summarised by means of count summaries by 
System Organ Class and Preferred Term assigned to the event. Other safety variables will be summarised as appropriate. The exploratory endpoints (resource utilisation and health-related quality of life) will be reported descriptively as part of SYGMA2.

In order to assess the consistency of the treatment effect in the two subgroups (defined by pre-study treatment), a pre-study treatment $\times$ treatment interaction term will be included in the models for the primary variables in SYGMA1 and SYGMA2. Several other subgroup analyses are also planned, including age, gender, severe exacerbation history in the 12 months prior to screening, baseline symptom history (ACQ), time since asthma diagnosis, smoking history, region, pre-bronchodilator $\mathrm{FEV}_{1}$, and SABA use during run-in.

\section{Discussion}

Both clinicians and patients may underestimate the risks and burden associated with mild asthma. Indeed, despite the proven efficacy of ICS maintenance therapy in asthma, there remains an unmet medical need due to over-reliance on SABA reliever medication and poor adherence to prescribed ICS [24]. Initial reports suggest that the as-needed use of combination ICS $/ \beta_{2}$-agonist with rapid onset of action may have advantages over regular ICS therapy for the treatment of mild asthma $[11,12]$. For example, in a 6-month trial of 455 patients with mild asthma, the symptom-driven use of $\mathrm{BDP} / \mathrm{sal}$ butamol was as effective for asthma exacerbations and morning PEF as maintenance $\mathrm{BDP}$ or $\mathrm{BDP} /$ salbutamol plus as-needed salbutamol [11], and significantly better than as-needed salbutamol.

Large randomised controlled trials investigating the asneeded use of different ICS/LABA combinations, taken in response to symptoms, as an alternative to current step 2 treatment in patients with mild asthma are currently lacking and are an important research priority $[15,16]$. SYGMA1 and SYGMA2 are the first major randomised controlled trials to assess the as-needed use of budesonide/ formoterol in patients with mild asthma. For SYGMA2, severe exacerbations are the primary outcome measure. However, for SYGMA1, well-controlled asthma weeks was chosen as the primary outcome measure due to its relevance to all patients. Consequently, the primary outcomes of the SYGMA studies address both aspects of the goals of asthma management, as defined by GINA and other treatment guidelines, which are to achieve good symptom control and to minimise the future risk of exacerbations.

Well-controlled asthma weeks has previously been used in several studies as a composite measure of asthma control $[19,25,26]$, but, to the best of our knowledge, this outcome measure has never been used in studies that include electronic monitoring of reliever use. Well-controlled asthma weeks takes into account symptoms, night-time awakenings, lung function, and as-needed reliever medication use, and corresponds to the 'symptom control' component in current guidelines, and excludes weeks with additional corticosteroid use or with night-time awakenings. However, the definition of well-controlled asthma weeks is based on current guidelines, where 'as needed' medication is usually a short-acting bronchodilator that does not contain an anti-inflammatory component, and so it may not be applicable to patients receiving as-needed budesonide/formoterol. Consequently, a sensitivity analysis will be performed that excludes the as-needed use of study medication from the definition of well-controlled asthma weeks. In addition, although patients are known to overreport their preventer use, they often under-report their reliever use [27]; as a result, there may be fewer wellcontrolled asthma weeks with reliever use recorded electronically than if this element had been based on selfreport. It should also be noted that the TUM is electronically recording the turning of the Turbuhaler ${ }^{\circ}$ grip, and not the actual process of inhalation, which may lead to over reporting of medication use.

A strength of the SYGMA studies is their long duration (52 weeks), which will allow reliable assessment of exacerbations. Reducing future risk by the prevention of asthma exacerbations is a key goal of asthma management [2] as exacerbations constitute the greatest risk to patients, are a cause of anxiety to patients and their families, and result in the greatest cost to the health care system [20]. There is some uncertainty over the frequency of severe exacerbations in mild asthma, with estimates ranging from 0.12 to 0.77 episodes per patient-year $[1,3,28]$. It has been suggested that severe exacerbations in mild asthma represent $30-40 \%$ of all asthma exacerbations that require an emergency consultation [1]. Twelve months is regarded as the minimum period for evaluation of annualised exacerbation rates [29]; this is particularly important in mild asthma, in which exacerbations are relatively rare events [30]. The designs of the SYGMA studies utilise clinic visits (SYGMA1 and SYGMA2), eDiary alerts (SYGMA1 only), and additional phone contacts (SYGMA2 only) to ensure that asthma exacerbation events are correctly collected.

The 52-week duration and use of an electronic monitoring device (TUM) on all blinded study inhalers will also allow evaluation of usage of the randomised maintenance and as-needed medications over all seasons of a year. Patients with mild asthma may be willing to accept mild symptoms and have poor adherence to maintenance ICS [31]. It is, therefore, hoped that the as-needed approach will better match patient behaviour and help to overcome the problems associated with poor adherence. Patients will be aware that inhaler use is being recorded, which may temporarily improve adherence with maintenance treatment; however, this is likely to occur only for a short period of time [32]. 
A number of other considerations have contributed to the design of the studies. For example, a 2-4-week run-in period has been included in both studies to allow baseline data to be collected and to ensure that patients are in need of GINA step 2 treatment. Furthermore, in SYGMA1 only, patient recruitment will be balanced to allow stratification based on pre-study treatment, i.e. asthma that is uncontrolled on as-needed short-acting bronchodilator or asthma that is well-controlled on mono-maintenance with either a low-dose ICS or LTRA plus as-needed short-acting bronchodilator. Health economic data will be collected in SYGMA2, to allow comparison of the cost-effectiveness of the different regimens under different pricing structures.

Data for the use of as-needed budesonide/formoterol compared with as-needed SABA in a similar patient population and in studies with a similar design to SYGMA are unavailable; as such, the SYGMA1 study includes a 'pseudo' placebo arm that allows the assessment of the superiority of as-needed budesonide/formoterol compared with as-needed terbutaline. However, patients in the 'pseudo' placebo arm may experience a deterioration in asthma control, and so it is important to ensure that there is a mechanism by which they receive rapid medical attention whenever necessary. Consequently, triggers in the eDiary generate alerts that warn patients of worsening asthma, prompting them to contact the investigator who may prescribe additional inhaled and/or systemic corticosteroid treatment to patients experiencing an exacerbation or with poor long-term asthma control. In addition, patients will be instructed to contact the investigator at any time should they require medical assistance or if they use more than 12 as-needed inhalations per day. The SYGMA2 study is designed to support the SYGMA1 regulatory study but reduce the burden of study requirements, e.g. fewer scheduled visits and absence of the eDiary/patient reminders. However, because of the regulatory requirements for a double-blind, placebo-controlled study, all participants will be required to take a regular maintenance inhaler containing either placebo or budesonide to ensure blinding of the treatment groups. Consequently, additional studies with a pragmatic, open-label design will be needed to evaluate natural patient behaviour with an as-needed regimen, so that the results can be generalised to clinical practice, and two such studies (Universal Trial Numbers: U1111-1170-2118; U1111-1174-2273) are underway [33].

In SYGMA 2, daily low-dose budesonide (plus as-needed terbutaline) was selected as the active comparator treatment. The use of regular daily low-dose budesonide is wellestablished, having been shown to be highly effective in reducing the risk of asthma-related exacerbations, including in patients with mild persistent asthma (as demonstrated in the START study) [3]. Furthermore, additional evidence has recently emerged confirming the appropriateness of low- dose budesonide maintenance therapy for patients with mild asthma and less frequent symptoms [34, 35].

Thus, the availability of an as-needed ICS/LABA single inhaler that could provide comparable control to current standard-of-care treatment with low-dose daily ICS plus asneeded medication would represent a breakthrough in the treatment of mild asthma, providing patients and clinicians with an alternative more convenient option with the potential for improved adherence. The blinded sample size review conducted during SYGMA 2 demonstrated adequate power to test a noninferiority hypothesis based on the design and assumptions specified in the study protocol. In view of this, the decision was taken to change the primary objective for SYGMA 2 to assess whether budesonide/formoterol as needed will be noninferior to budesonide given as regular maintenance with regard to the annual severe exacerbation rate. To maintain the validity of the primary analysis, the protocol was amended prior to trial completion and unblinding, and the noninferiority margin relative to the control arm was pre-specified [36, 37].While a noninferiority margin for exacerbation reduction in patients with mild asthma has not been previously defined, a margin of $20 \%$ was considered to be an appropriate choice because a difference between treatments smaller than this would be judged to be of questionable clinical relevance in this population. If the noninferiority criteria are met in SYGMA 2, superiority of as-needed budesonide/formoterol versus budesonide will also be assessed. The benefit/risk will also be assessed in the context of other endpoints evaluating asthma control as well as steroid load, the pattern of 'as needed use' and controller use days.

In conclusion, the SYGMA programme should help to determine the efficacy and safety of as-needed budesonide/formoterol combination therapy in mild asthma, as an alternative to regular low-dose ICS treatment. Patient recruitment is completed and completion of the phase 3 studies is planned in 2017.

\section{Trial status}

The first patients were recruited to SYGMA1 and SYGMA2 in July 2014 and November 2014, respectively. Recruitment to both trials is completed (June 2016).

\section{Additional files}

Additional file 1: Table S1. Oversight Authorities for SYGMA1 and SYGMA2. (DOCX $20 \mathrm{~kb}$ )

Additional file 2: SPIRIT Checklist for SYGMA1 and SYGMA2. (DOCX 52 kb)

\section{Abbreviations}

ACQ-5: Asthma Control Questionnaire 5-item version; AE: Adverse event; ANCOVA: Analysis of covariance; AQLQ: Standardised Asthma Quality of Life Questionnaire; ATS: American Thoracic Society; bid: Twice daily; BDP: Beclometasone dipropionate; Cl: Confidence interval; eCRF: Electronic Case Report Form; ED: Emergency department; eDiary: Electronic diary; 
ePRO: Electronic Patient Reported Outcome; ERS: European Respiratory Society; FeNO: Fractional exhaled nitric oxide; FEV : Pre-dose forced expiratory volume in $1 \mathrm{~s}$; GCS: Glucocorticosteroid; GINA: Global Initiative for Asthma; HR: Hazard ratio; ICS: Inhaled corticosteroid; LABA: Long-acting $\beta_{2-}$ agonist; LSM: Least squared mean; LTRA: Leukotriene receptor antagonist; PEF: Peak expiratory flow; SABA: Short-acting $\beta_{2}$-agonist; SAE: Serious adverse event; SYGMA: SYmbicort Given as needed in Mild Asthma; TUM: Turbuhaler Usage Monitor

\section{Acknowledgements}

Clair Clowes and Katharine Williams from inScience Communications, Springer Healthcare provided medical writing assistance, funded by AstraZeneca.

\section{Funding}

AstraZeneca funded the SYGMA programme and collaborated with researchers in the design and planned statistical analyses.

\section{Availability of data and materials}

More information on AstraZeneca's clinical trials disclosure policy is available at: http://astrazenecagrouptrials.pharmacm.com//ST/Submission/Disclosure A completed SPIRIT Checklist is available in Additional file 2.

\section{Authors' contributions}

PMOB, EB, JMF, and HKR proposed the SYGMA programme in 2008, and PMOB, $J M F, N Z, E B, P J B, C K, G A, K P, C J, S I$, and HKR provided input into the concept and design of the studies. PMOB and EB are the international coordinating investigators for SYGMA1 and SYGMA2, respectively, and NZ and JMF are principal investigators. All authors helped draft each stage of the manuscript, and read and approved the final version at the time of submission.

\section{Competing interests}

Paul M O'Byrne has received compensation for board membership from the Joint Oversight Board for a LABA safety study; has received consultancy or speaker's fees from AstraZeneca, Boehringer Ingelheim, Chiesi, GlaxoSmithKline, Merck, and Novartis; has received or has grants pending from Ambit, Amgen, AstraZeneca, Axikin, Genentech, and Novartis; and is a member of the Global Initiative for Asthma (GINA) Science Committee. J Mark FitzGerald has served on advisory boards for Aerocrine, AstraZeneca, Boehringer Ingelheim, GlaxoSmithKline, Merck, Novartis, Pfizer, and TEVA; has been a member of speakers' bureaus for ALK, AstraZeneca, Boehringer Ingelheim, GlaxoSmithKline, Merck, Novartis, and Pfizer; and is a member of the Global Initiative for Asthma (GINA), is chair of the GINA Executive (until 31 December 2015, after which he will remain a member of the Executive), and a member of its Science Committee.

Nanshan Zhong has served on advisory boards for AstraZeneca, Boehringer Ingelheim, GlaxoSmithKline, Novartis, and Pfizer; has been a member of speakers' bureaus for AstraZeneca, Boehringer Ingelheim, GlaxoSmithKline, Novartis, and Pfizer; and has received consultancy fees from AstraZeneca, Boehringer Ingelheim, GlaxoSmithKline, Merck, Novartis, and Pfizer. Eric Bateman has received consulting fees from AstraZeneca for participation in a working group; has received compensation for board membership from Almirall, AstraZeneca, Boehringer Ingelheim, Elevation Pharma, Forest, GlaxoSmithKline, Merck, Novartis, and Nycomed; consultancy fees from ALKAbelló, Almirall, and Cephalon; his institution has received grants from Actelion, Aeras, Almirall, AstraZeneca, Boehringer Ingelheim, Cephalon, Forest, GlaxoSmithKline, Roche/Genentech, Merck, Novartis, Takeda, and TEVA; and he has received payment for delivering lectures from AstraZeneca, Boehringer Ingelheim, Chiesi, GlaxoSmithKline, Novartis, Pfizer, and Takeda, as well as from Novartis for the development of education presentations.

Peter Barnes has served on Scientific Advisory Boards for AstraZeneca, Boehringer Ingelheim, Chiesi, Daiichi-Sankyo, GlaxoSmithKline, Novartis, Nycomed, Pfizer, Teva, and UCB; has received research funding from Aquinox Pharmaceuticals, AstraZeneca, Boehringer-Ingelheim, Chiesi, Daiichi-Sankyo, GlaxoSmithKline, Novartis, Nycomed, Pfizer, and Prosonix; and is a cofounder of RespiVert (now part of Johnson \& Johnson), which has discovered novel inhaled anti-inflammatory treatments for asthma and COPD. Helen Reddel has received consultancy fees from AstraZeneca for face-toface meetings; has received compensation for board membership from AstraZeneca, Boehringer Ingelheim, GlaxoSmithKline, Merck, and Novartis; has received consultancy fees from AstraZeneca and Novartis; has received grants from AstraZeneca and GlaxoSmithKline; has received payment from
AstraZeneca, Boehringer Ingelheim, GlaxoSmithKline, Novartis, Mundipharma, and Teva for delivering independent educational presentations; and is Chair of the Global Initiative for Asthma (GINA) Science Committee. Christina Keen, Gun Almqvist, Kristine Pemberton, Carin Jorup, and Stefan Ivanov are all employees of AstraZeneca.

\section{Consent for publication}

All of the authors have provided consent for the publication of the manuscript, and all associated materials.

\section{Ethics approval and consent to participate}

Ethical oversight authorities for SYGMA

Australia: Department of Health and Ageing Therapeutic Goods Administration, Human Research Ethics Committee; Brazil: National Committee of Ethics in Research, National Health Surveillance Agency; Bulgaria: Bulgarian Executive Drug Agency, Ethics Committee for Multicentre Studies: Canada: Health Canada; Chile: Instituto de Salud Pública; China: State Food and Drug Administration; Hungary: National Institute of Pharmacy, Research Ethics Medical Committee; Korea: Ministry of Food and Drug Safety; Mexico: Comité Bioético para la Investigación Clínica, Comité de Ética en Investigación de la Facultad de Medicina y Hospital Universitario, Federal Commission for Protection Against Health Risks; Peru: Instituto Nacional de Salud; Phillippines: Bureau of Food and Drugs; Poland: Komisja Bioetyczna Przy Okręgowej Izbie Lekarskiej W Białymstoku, Office for Registration of Medicinal Products, Medical Devices and Biocidal Products; Romania: National Medicines and Medical Devices Agency; Russia: Ethics Committee of the Ministry Of Health of the Russian Federation, Ministry of Health of the Russian Federation; South Africa: Medicines Control Council; Ukraine: Central Ethics Committee of the Ministry of Health of Ukraine, State Pharmacological Center - Ministry of Health; United Kingdom: Medicines and Healthcare Products Regulatory Agency; Vietnam: Ministry of Health Ethical oversight authorities for SYGMA 2

Australia: Department of Health and Ageing Therapeutic Goods Administration, Human Research Ethics Committee; Brazil: National Committee of Ethics in Research, National Health Surveillance Agency; Bulgaria: Bulgarian Executive Drug Agency, Ethics Committee for Multicentre Studies; Chile: Instituto de Salud Pública; Colombia: Ministry of Health, National Institute of Food and Drug Monitoring; Czech Republic: State Institute for Drug Control; France: National Agency for the Safety of Medicines and Health Products; Germany; Federal Institute for Drugs and Medical Devices, Ministry of Health, Paul Erlich Institute sin Langen; Hungary: National Institute of Pharmacy, Research Ethics Medical Committee; Mexico: Comité Bioético para la Investigación Clínica, Comité de Ética en Investigación de la Facultad de Medicina y Hospital Universitario, Federal Commission for Protection Against Health Risks; New Zealand: Medicines and Medical Devices Safety Authority, New Zealand Ministry of Health, PHARMAC; Peru: Instituto Nacional de Salud; Phillippines: Bureau of Food and Drugs; Poland: Komisja Bioetyczna przy Wielkopolskiej Izbie Lekarskiej, Urzad Rejestracji Produktow Leczniczych Wyrobow Medycznych i Produktow Biobojczych; Romania: National Medicines and Medical Devices Agency; Russia: Ethics Committee of the Ministry Of Health of the Russian Federation, Ministry of Health of the Russian Federation; Saudi Arabia: National Committee for Medical and Bio-ethics, King Abdulaziz City for Science and Technology, Saudi Food and Drug Authority Contact; Slovakia: Etika Komisa Urad Kosickeho Samospravneho Kraja, State Institute for Drug Control/Ministry of Health; South Africa: Medicines Control Council; South Korea: Ministry of Food and Drug Safety; Spain: Spanish Agency for Medicines and Health Products; Sweden: Medical Products Agency; Thailand: Ministry of Public Health; Ukraine: Central Ethics Committee of the Ministry of Health of Ukraine, State Pharmacological Center - Ministry of Health; Vietnam: Ministry of Health Patients provided informed consent before enrolling in the studies, and were free to withdraw consent at any time.

\section{Author details}

${ }^{1}$ Michael G DeGroote School of Medicine, Faculty of Health Sciences, McMaster University, Hamilton, ON, Canada. ${ }^{2}$ Institute for Heart and Lung Health, University of British Columbia, Vancouver, BC, Canada. ${ }^{3}$ State Key Laboratory of Respiratory Diseases, First Affiliated Hospital, Guangzhou Medical University, Guangzhou, China. ${ }^{4}$ Department of Medicine, University of Cape Town, Cape Town, South Africa. ${ }^{5}$ Airway Disease Section, National Heart and Lung Institute, Imperial College, London, UK. ${ }^{6}$ AstraZeneca R\&D, 
Gothenburg, Sweden. ${ }^{7}$ AstraZeneca, Alderley Park, Macclesfield, UK. ${ }^{8}$ Clinical Management Group, Woolcock Institute of Medical Research, University of Sydney, Sydney, Australia. ${ }^{9}$ Firestone Institute of Respiratory Health, St Joseph's Healthcare and Department of Medicine, McMaster University, 1280 Main Street West, Hamilton, ON L8S 4K1, Canada.

Received: 21 March 2016 Accepted: 28 November 2016

Published online: 10 January 2017

\section{References}

1. Dusser D, Montani D, Chanez P, de Blic J, Delacourt C, Deschildre A, et al. Mild asthma: an expert review on epidemiology, clinical characteristics and treatment recommendations. Allergy. 2007;62(6):591-604. doi:10.1111/j.13989995.2007.01394.x.

2. Global Strategy for Asthma Management and Prevention. Updated 2015. Global Initiative for Asthma. 2015. http://ginasthma.org/wp-content/ uploads/2016/01/GINA_Report_2015_Aug11-1.pdf. Accessed 13 Oct 2015.

3. Pauwels RA, Pedersen S, Busse WW, Tan WC, Chen YZ, Ohlsson SV, et al. Early intervention with budesonide in mild persistent asthma: a randomised, double-blind trial. Lancet. 2003;361(9363):1071-6. doi:10.1016/S01406736(03)12891-7.

4. Patel M, Pilcher J, Pritchard A, Perrin K, Travers J, Shaw D, et al. Efficacy and safety of maintenance and reliever combination budesonide-formoterol inhaler in patients with asthma at risk of severe exacerbations: a randomised controlled trial. Lancet Respir Med. 2013;1(1):32-42. doi:10.1016/S2213-2600(13)70007-9.

5. Barnes CB, Ulrik CS. Asthma and adherence to inhaled corticosteroids: current status and future perspectives. Respir Care. 2015;60(3):455-68. doi: 10.4187/respcare.03200.

6. Suissa S, Ernst P, Benayoun S, Baltzan M, Cai B. Low-dose inhaled corticosteroids and the prevention of death from asthma. N Engl J Med. 2000;343(5):332-6. doi: 10.1056/NEJM200008033430504

7. Why asthma still kills: The National Review of Asthma Deaths (NRAD). Royal College of Physicians. 2014. https://www.rcplondon.ac.uk/file/868/ download?token=3wikiuFg. Accessed 13 Oct 2015.

8. Suissa S, Ernst P, Kezouh A. Regular use of inhaled corticosteroids and the long term prevention of hospitalisation for asthma. Thorax. 2002;57(10):880-4.

9. Williams LK, Peterson EL, Wells K, Ahmedani BK, Kumar R, Burchard EG, et al. Quantifying the proportion of severe asthma exacerbations attributable to inhaled corticosteroid nonadherence. J Allergy Clin Immunol. 2011;128(6): 1185-91.e2. doi:10.1016/j.jaci.2011.09.011.

10. Rabe KF, Adachi M, Lai CK, Soriano JB, Vermeire PA, Weiss KB, et al. Worldwide severity and control of asthma in children and adults: the global asthma insights and reality surveys. J Allergy Clin Immunol. 2004;114(1):40-7. doi:10.1016/j.jaci. 2004.04.042.

11. Papi A, Canonica GW, Maestrelli P, Paggiaro P, Olivieri D, Pozzi E, et al. Rescue use of beclomethasone and albuterol in a single inhaler for mild asthma. N Engl J Med. 2007;356(20):2040-52. doi:10.1056/NEJMoa063861.

12. Calhoun WJ, Ameredes BT, King TS, Icitovic N, Bleecker ER, Castro M, et al. Comparison of physician-, biomarker-, and symptom-based strategies for adjustment of inhaled corticosteroid therapy in adults with asthma: the BASALT randomized controlled trial. JAMA. 2012;308(10):987-97. doi:10. 1001/2012.jama.10893.

13. Haahtela T, Tamminen K, Malmberg LP, Zetterstrom O, Karjalainen J, YlaOutinen $\mathrm{H}$, et al. Formoterol as needed with or without budesonide in patients with intermittent asthma and raised NO levels in exhaled air: a SOMA study. Eur Respir J. 2006;28(4):748-55. doi:10.1183/09031936.06.00128005.

14. Rabe KF, Atienza T, Magyar P, Larsson P, Jorup C, Lalloo UG. Effect of budesonide in combination with formoterol for reliever therapy in asthma exacerbations: a randomised controlled, double-blind study. Lancet. 2006; 368(9537):744-53. doi:10.1016/S0140-6736(06)69284-2.

15. Papi A, Caramori G, Adcock IM, Barnes PJ. Rescue treatment in asthma. More than as-needed bronchodilation. Chest. 2009;135(6):1628-33. doi:10. 1378/chest.08-2536.

16. Beasley R, Weatherall M, Shirtcliffe P, Hancox R, Reddel HK. Combination corticosteroid/beta-agonist inhaler as reliever therapy: a solution for intermittent and mild asthma? J Allergy Clin Immunol. 2014;133(1):39-41. doi:10.1016/j.jaci.2013.10.053.

17. Global Strategy for Asthma Management and Prevention. Updated 2012. Global Initiative for Asthma. 2012. http://www.ginasthma.org/local/uploads/ files/GINA_Report_March13_1.pdf. Accessed 13 Oct 2015.
18. Gradinarsky L, Lööf T, editors. Inhalation adherence monitoring using smart electronic add-on device. MobiHealth: 4th International Conference on Wireless Mobile Communication and Healthcare - 'Transforming healthcare through innovations in mobile and wireless technologies'. Athens: European Union Digital Library; 2014.

19. Bateman ED, Boushey HA, Bousquet J, Busse WW, Clark TJ, Pauwels RA, et al. Can guideline-defined asthma control be achieved? The Gaining Optimal Asthma ControL study. Am J Respir Crit Care Med. 2004;170(8):836-44. doi: 10.1164/rccm.200401-0330C

20. Reddel HK, Taylor DR, Bateman ED, Boulet LP, Boushey HA, Busse WW, et al. An official American Thoracic Society/European Respiratory Society statement: asthma control and exacerbations: standardizing endpoints for clinical asthma trials and clinical practice. Am J Respir Crit Care Med. 2009; 180(1):59-99. doi:10.1164/rccm.200801-060ST.

21. Miller MR, Hankinson J, Brusasco V, Burgos F, Casaburi R, Coates A, et al. Standardisation of spirometry. Eur Respir J. 2005;26(2):319-38. doi:10.1183/ 09031936.05.00034805.

22. Pilcher J, Shirtcliffe P, Patel M, McKinstry S, Cripps T, Weatherall M, et al. Threemonth validation of a turbuhaler electronic monitoring device: implications for asthma clinical trial use. BMJ Open Resp Res. 2015:2:e000097.

23. Barbour RS. The role of qualitative research in broadening the 'evidence base' for clinical practice. J Eval Clin Pract. 2000;6(2):155-63.

24. O'Byrne P, Cuddy L, Taylor DW, Birch S, Morris J, Syrotuik J. Efficacy and cost benefit of inhaled corticosteroids in patients considered to have mild asthma in primary care practice. Can Respir J. 1996;3(3):169-75.

25. NCT00463866. Local Phase 4 Pan-European SMART Study. 2015. https:// www.clinicaltrials.gov/ct2/show/NCT00463866. Accessed 16 June 2015.

26. NCT00315744. Viapaed Study in children and adolescents with asthma. 2015. https://www.clinicaltrials.gov/ct2/show/NCT00315744. Accessed 16 June 2015.

27. Patel $M$, Perrin $K$, Pritchard $A$, Williams $M$, Wijesinghe $M$, Weatherall $M$, et al. Accuracy of patient self-report as a measure of inhaled asthma medication use. Respirology. 2013;18(3):546-52. doi:10.1111/resp.12059.

28. O'Byrne PM, Barnes PJ, Rodriguez-Roisin R, Runnerstrom E, Sandstrom T, Svensson $\mathrm{K}$, et al. Low dose inhaled budesonide and formoterol in mild persistent asthma: the OPTIMA randomized trial. Am J Respir Crit Care Med. 2001:164(8 Pt 1):1392-7. doi:10.1164/ajrccm.164.8.2104102.

29. Fuhlbrigge A, Peden D, Apter AJ, Boushey HA, Camargo Jr CA, Gern J, et al. Asthma outcomes: exacerbations. J Allergy Clin Immunol. 2012;129(3 Suppl): S34-48. doi:10.1016/j.jaci.2011.12.983.

30. de Vries F, Setakis E, Zhang B, van Staa TP. Long-acting \{beta\}2-agonists in adult asthma and the pattern of risk of death and severe asthma outcomes: a study using the GPRD. Eur Respir J. 2010;36(3):494-502. doi:10.1183/ 09031936.00124209

31. Shahidi N, Fitzgerald JM. Current recommendations for the treatment of mild asthma. J Asthma Allergy. 2010;3:169-76. doi:10.2147/JAA.S14420.

32. Foster JM, Smith L, Bosnic-Anticevich SZ, Usherwood T, Sawyer SM, Rand CS, et al. Identifying patient-specific beliefs and behaviours for conversations about adherence in asthma. Intern Med J. 2012;42(6):e136-44. doi:10.1111/j.1445-5994.2011.02541.x.

33. Beasley R, Pavord I, Papi A, Reddel HK, Harrison T, Marks G et al. Description of randomised controlled trial of ICS/LABA reliever therapy in mild asthma. Eur Respir J. 2015. In press.

34. Reddel HK, Busse WW, Pedersen S, Tan WC, Chen Y-Z, Jorup C, et al. Should recommendations about starting inhaled corticosteroid treatment for mild asthma be based on symptom frequency: a post-hoc efficacy analysis of the START study. Lancet. 2016. doi:10.1016/S01406736(16)31399-X

35. Global Strategy for Asthma Management and Prevention. Updated 2016. Global Initiative for Asthma. 2016. http://ginasthma.org/2016-gina-report-global-strategyfor-asthma-management-and-prevention/. Accessed 7 Oct 2016

36. CPMP. Committee for Proprietary Medicinal Products. London; 2000. http:// www.ema.europa.eu/docs/en_GB/document_library/Scientific_guideline/ 2009/09/WC500003658.pdf. Accessed 7 Oct 2016.

37. Pocock SJ, Stone GW. The primary outcome fails-What next? N Engl J Med. 2016:375(9):861-70. doi:10.1056/NEJMra1510064.

38. Quanjer PH, Stanojevic S, Cole TJ, Baur X, Hall GL, Culver BH, et al. Multiethnic reference values for spirometry for the 3-95-yr age range: the global lung function 2012 equations. Eur Respir J. 2012;40(6):1324-43. doi:10.1183/ 09031936.00080312 